\title{
A Review on Multi-Robot Systems Categorised by Application Domain*
}

\author{
Rachael N. Darmanin ${ }^{1}$ and Marvin K. Bugeja ${ }^{1}$
}

\begin{abstract}
Literature reviews on Multi-Robot Systems (MRS) typically focus on fundamental technical aspects, like coordination and communication, that need to be considered in order to coordinate a team of robots to perform a given task effectively and efficiently. Other reviews only consider works that aim to address a specific problem or one particular application of MRS. In contrast, this paper presents a survey of recent research works on MRS and categorises them according to their application domain. Furthermore, this paper compiles a number of seminal review works that have proposed specific taxonomies in classifying fundamental concepts, such as coordination, architecture and communication, in the field of MRS.
\end{abstract}

\section{INTRODUCTION}

Since the late 1980s researchers have been motivated to design and build teams of robots with the ability of working together on some given task. This motivation stems from the fact that in many applications, Multi-Robot Systems (MRS) bring about several advantages over Single Robot Systems. In particular, MRS are generally more time-efficient, less prone to single-points of failure, and typically exhibit multiple capabilities, which in many cases yield a more effective solution to a given problem. In early works, researchers observed natural systems, such as a swarm of bees, ants and even humans, to study how a group of individual entities can work together to perform a given task. The multidisciplinary nature of these early studies, eventually led to MRS being applied in several different application domains such as surveillance, search and rescue, foraging, exploration, cooperative manipulation, and transportation of objects, among others. This paper reviews a number of prominent and recent research works that aim to address various problems appertaining to six main application domains of MRS.

In Section II we compile a number of previous surveys on MRS and categorise them in six broad categories, while in Section III we present a number of recent research works on MRS and organise them according to their application domain. We believe that this new categorisation can be very useful to researchers who are mainly interested in one particular domain. Furthermore, this survey can also help promote the migration of ideas across different application domains of MRS. Finally in Section IV, we draw a number of conclusions about the current and most prominent challenges in the field of MRS.

\footnotetext{
*The research work disclosed in this publication is partially funded by the ENDEAVOUR Scholarships Scheme 2014-2020, that may be part-financed by the European Union.

${ }^{1}$ Rachael N. Darmanin and Marvin K. Bugeja are with the Department of Systems and Control Engineering, Faculty of Engineering, University of Malta, MSD2080, Malta \{rachael.darmanin, marvin.bugeja\}@um.edu.mt
}

\section{RELATED WORK}

The majority of literature review papers on Multi-Robot Systems (MRS) focus on classifying the most fundamental aspects of an MRS, such as coordination and communication. In [1] Farinelli et al. classify these MRS features into two dimensions. The first, termed the coordination dimension, deals with the different classes of cooperation schemes, such as whether the system is centralized or decentralized, strongly cooperative (i.e. following a strict protocol), or weakly cooperative, among others. The system dimension classifies the existing types of communication schemes and team decomposition attributes. Similarly, in [2] Parker classifies MRS according to their architecture, the heterogeneity in the team, the type of communication scheme adopted, and the different types of task allocation schemes. In this work, Parker also briefly reviews some works according to their application domain. However, the latter is not an extensive literature review of such works. A similar reviewing approach that categorises works based on the foundation topics of MRS, namely coordination, task allocation and cooperation, is also adopted in [3]-[5]. Furthermore, in contrast to these works, in [6] Gerkey and Mataric focus on one particular aspect of MRS, namely Multi-Robot Task Allocation (MRTA), and propose a taxonomy of task allocation schemes. The same taxonomy is also used in [7].

Alternatively, a literature review may also focus on particular schemes forming part of the main components in an MRS. In [8], Bernardine Dias et al. focus on classifying what they call market-based coordination approaches, which is a type of MRTA scheme. Such coordination schemes require the robots to bid for the tasks that they are able to perform. Furthermore, detailed reviews in the area of swarm robotics are properly surveyed in [9]-[11], while those reviewing biologically-inspired research include [12]. We encourage the interested reader to refer to these works for more details.

In recent years, considerable attention has been given to human-robot interaction (HRI). In this light, Goodrich et al. [13] provide an extensive review of the history of HRI, as well as a review of works that introduced HRI to the field of MRS. In this work the author identifies the main topics in the field of HRI and provides a list of challenges that could shape the future of this interesting research area. Similarly, Chen et al. [14], propose a review of the human factors in systems exhibiting HRI, focussing on human supervision of multiple robots, and maintaining the human operator's situation awareness while retaining authority in the decisionmaking process. Both Goodrich et al. and Chen et al. provide indicators to the current open challenges in this area. 
Moreover, some review papers focus on specific problems in MRS, such as formation control of robots in a team. Guanghua et al. [15] review such works in light of the type of formation architecture, and the existing formation control strategies employed. In the latter classification the authors classify the reviewed approaches according to the following categories: behaviour-based approaches, leader-follower approaches, virtual structure approaches, artificial potential functions or graph-based approaches. In [16], the authors review the mathematical problem of flocking, together with existing flocking control strategies. Other problem-specific reviews focus on patrolling algorithms [17], robotic urban search and rescue [18], and autonomous underwater vehicles [11], [19]. The review papers referred to in this section have been grouped in six broad categories in Table I. In contrast to the survey works mentioned in this section, this paper's contribution lies in a review of recent and notable works in MRS categorised according to the most prominent application domains of the field.

\section{APPLICATION DOMAINS}

During the years a number of general application domains in Multi-Robot Systems (MRS) have been proposed. For instance, both Parker [2] and Farinelli et al. [1] list the main application domains in the field of MRS. However, the aim of these works is not to review prominent and recent applications of MRS, but rather to provide a review of the main technical aspects and challenges of the field. The scope of this section is to address this gap, and classify recent works on MRS according to their application domains.

\section{A. Surveillance and, Search and Rescue}

Surveillance and, search and rescue applications have attracted considerable attention from the MRS community over the years. This is due to the relevance of such applications in daily life. Surveillance applications were initially reserved to patrolling or surveying indoor areas [21]. However, with the introduction of unmanned aerial vehicles (UAVs), researchers have expanded their study to include the surveillance of outdoor areas, such as areas far out at sea [22]. One of the main challenges in these applications is that of persistent surveillance due to the fact that one-time coverage and exploration algorithms cannot be used directly to continuously patrol and monitor the same area [23]. Nigam et al. [23] propose a novel control policy for persistent surveillance that maintains optimal performance through a formally-derived and scalable heuristic method. In this work, the environment

TABLE I

SUMMARY OF REVIEW PAPERS

\begin{tabular}{|l||c|}
\hline MRS Main Principles & {$[1]-[8]$} \\
\hline Swarm Robotics & {$[9]-[11]$} \\
\hline Biologically-Inspired Works & {$[12],[20]$} \\
\hline Human-Multi-Robot Interaction & {$[13],[14]$} \\
\hline Problem-Specific Works & {$[15]-[18]$} \\
\hline Autonomous Underwater Vehicles & {$[11],[19]$} \\
\hline
\end{tabular}

is divided into grid cells, where each cell is attributed with an age and the goal is to minimize the overall age of all the cells. A control policy called the Multi-Agent Reactive Policy is proposed to control the UAVs performing surveillance. A similar approach is adopted in [24] where the authors solve the task of persistent surveillance through the Vehicle Routing Problem.

Task allocation is another challenge in such applications because the solution to this problem must be time-efficient. In [25], the authors make use of a market-based strategy where the robots bid for locations that must be surveyed. In contrast, Jeon et al. [26] calculate costs for a set of tasks, and allocate the mission tasks to the robots according to these costs.

Over the years disasters such as the Fukushima nuclear accident in 2011 have enabled researchers to deploy advanced MRS in real-life applications, mostly for search and rescue. For example, in [22] the authors propose a cooperative scheme for a multi-robot team for the surveillance of shipwreck survivors at sea. Using satellite imagery the user can plan the mission waypoints, which are then followed by an Unmanned Surface Vehicle (USV) carrying a UAV. When the USV arrives at the designated way-point, the UAV takes off and uses a grid-like search pattern and image processing to localize survivors.

Another example is that of Gregory et al. [27], who address a humanitarian assistance and disaster relief applicaton. In this work the authors focus on simultaneously evaluating the damage done to the environment, and localising the victims according to two types of goals, namely, goals established from prior maps, and dynamic goals established according to the sudden detection of victims. The novelty of this work lies in addressing unreliable autonomy and communication by modelling unknown travel costs in a dynamic variant of the Capacitated Team Orienteering Problem [28].

Moreover, in [29], the authors also address the humanitarian relief problem through an implementation of a heterogeneous robotic system. This system features land and marine mobile stations that are responsible for coordinating and supporting UAVs and fast-speed land or marine robots. The authors propose solutions to two coordination technical challenges. The first entails the localization and landing of the UAVs - achieved through the use of visual SLAMand UAV battery replacement to mitigate the limited energy constraint, which is a very common problem in MRS. In this work the authors also propose a collaboration scheme for the team of UAVs based on dynamic communication, target identification and triangulation.

\section{B. Foraging and Flocking}

The task of foraging is often synonymous with swarm robotics, which is inspired by natural colonial systems such as those of bees and ants. This is because very often, the decentralized team only requires implicit communication to cooperatively collect randomly distributed objects and transport them to a "home" location. In [30] Parker proposes ALLIANCE, a fault-tolerant framework that assigns tasks 
to robots according to their motivation and capabilities modelled through a behaviour set-to do these tasks. This framework was applied to the foraging task of cleaning up hazardous waste, where the task allocation and coordination among the team involved assigning the robots to move the waste or report back to the base station. In another behaviourbased approach [31], Schneider-Fontán and Matarić assign segments from a territory to each robot for clean-up and object collection, as opposed to the task assignment used in [30]. One challenge in this domain is the optimal sharing of navigational space. In [32] Lein and Vaughan propose an algorithm that reduces mutual spatial interference and exploits a non-uniform distribution of robots during foraging. To achieve this, the authors propose a technique named adaptive bucket brigade foraging, where the robots remain within a variable space distribution within the environment. Furthermore, since foraging is a task associated with natural systems, a number of works adopt particle swarm optimization (PSO). Particularly, Couceiro et al. [33] study the robotic Darwinian PSO under communication constraints in the team [33]. From these works one can conclude that in general, foraging is not implemented using complex explicit communication schemes. The preferred choice of architecture is often decentralized, in order to allow the team members to achieve the task with minimal interference between them.

The task of flocking, also called shepherding, is considered to be a trait of swarm robotics. Some works make use of behaviour-based models and the generation of safe zones, such that the members in the MRS can follow a direction and stay in line with the flock to maintain cohesion, but at the same time maintain enough distance between them to avoid collision [34]. In these cases, a decentralized architecture is often adopted. Moreover, in [35] Sakai et al. propose a novel flocking algorithm that treats all detected objects as obstacles, irrespective of whether they are truly obstacles or form part of the team. They argue that such a method limits the amount of information handled by the team since velocity information on neighbouring robots is not required. On the contrary, in [36] $\mathrm{Gu}$ and Wang propose a leaderfollower flocking technique, which requires the followers to communicate with their neighbours to exchange information about the estimated position of the flocking centre. Moreover, in [37] the authors apply reinforcement learning together with flocking control to enable a decentralized MRS to learn how predators should be avoided while maintaining the connectivity required for flocking. Additionally, in [38] the authors propose a control algorithm that allows a flock to navigate around obstacles. Similar to foraging, in flocking we can see a trend in the use of a decentralized architecture. However, the complexity of the task increases in flocking applications since the team must not only coordinate to avoid spatial interference, but also to maintain connectivity among the entities.

\section{Formation and Exploration}

In formation applications, the team of robots must maintain some strict arrangement while at the same time avoid obstacles in its path. This problem becomes more complex than flocking since an obstacle must be collectively avoided without any of the team members leaving the formation for a long while. A common solution is the leader-follower approach where a trajectory-planning algorithm is implemented on the leader robot and formation constraintsdistances from the leader-restrict the followers to maintain formation around the leader [39]. Recent research is also adopting computational intelligence methods in formation applications. In [40], Wang et al. solve the optimal formation problem by using a recurrent neural network. Shape theory is used to generate a set of feasible formations and the proposed optimal formation solution chooses the one that has the minimum distance from the initial formation. Alternatively, the work reported in [41] adopts fuzzy logic to achieve formation control. Additionally, the work in [42] adopts control schemes, such as Model Predictive Control, in order to establish formation in the team.

In contrast to formation, in exploration the robots in a team must distribute themselves in an unknown environment in order to explore the area. The coordination of such a system brings about many challenges, particularly those related to connectivity and battery-life problems. In [43] Banfi et al. address the problem of communication constraint by proposing an exploration strategy under recurrent connectivity. The robots only need to connect to the base station if new observations are made, hence allowing the members to disconnect for long periods of time until new information is obtained. Moreover, Cesare et al. [44] address the problems of communication and battery life. They propose a statebased approach in which a robot explores and shares information only if it is within the communication range and its battery levels are above a certain threshold. If the robot does not have enough energy to continue exploring, it waits for another robot to meet it, and then uses its remaining energy to relay the information to base. This rendezvous solution to overcome communication limitations is also used in [45].

Other works in this domain focus on exploration strategies that are tailor-made for a particular MRS. For instance, in [46] the authors propose a circle partitioning method that segments the environment into sections and assigns each robot to a particular sub-region. In [47] the authors use a flooding algorithm that aims at reducing the exploration time and minimize the overall distance travelled by the robots during exploration. A number of works even propose exploration strategies that emerge from a graph-based approach, such that optimal coordination can be achieved when having a known number of robots exploring an area [48].

\section{Cooperative Manipulation}

The box-pushing problem has become synonymous with MRS and it has been studied in several early works [49][51]. In one of these works, namely that by Brown and Jennings [52], the authors implement a pusher/steerer system where the object is moved from one place to another by small mobile robots. The steerer robot is pre-programmed with a trajectory and the pusher robot exerts a force onto the 
object, such that the steerer can follow its programmed path by setting its heading. Hence, communication is evidently absent from such a system. Alternatively, in [53] Sieber et al. propose a novel linear state feedback controller to surround an object with a formation of robots in order to transport said object. In this work the authors propose a suboptimal control law similar to the linear quadratic regulator (LQR) approach, which is used to regulate the way that a group of robots form an assembly around an object to transport it. Other works which adopt this formation control for object manipulation and transportation include [54] and [55].

More recently, Amanatiadis et al. [56] propose the system AVERT which is used to extract and transport vehicles from a specific location. The novelty of this work is focussed on lifter mobile robots used in a system that applies the concept of trajectory planning (using the $\mathrm{D}^{*}$ Lite method), obstacle detection, and makes use of intercommunication in order to exchange control and trajectory information with a command base. As opposed to the previously mentioned works, the approach proposed in the AVERT project requires a stable communication among the team members, which is often missing in traditional pusher-steerer or formationbased cooperative manipulation algorithms. In view of the solutions presented in these works, one may also think of foraging as another solution to cooperative object transportation and manipulation, since this involves the collection and transportation of items to some base location.

\section{E. Team Heterogeneity}

Heterogeneity in a team of mobile robots enables the team to handle complex tasks more efficiently and effectively by exploiting the benefits of the diverse capabilities of its members. In this work, we shall analyse heterogeneity from two perspectives, namely, human-robot heterogeneity and heterogeneous robot teams.

A human-robot interaction (HRI) may be present in a system where the human is supervising and commanding a team of multiple robots. In [57] Rossi et al. propose a scheme where the human operator communicates with a whole team of robots to assign tasks and specify the members which must perform each task through speech utterances. In this case this study focuses on how speech may be segmented to simultaneously address multiple robotic recipients. Similarly, Cacace et al. [58] apply an HRI to a search and rescue application. In their proposed scheme they exploit human gestures and speech to select a desired robot for a task in a nonverbal and implicit manner. Robot selection is estimated by a probability that a particular command given by the user infers a set of capabilities which the robot possesses. For instance, the command "take off" shall probably be directed to an aerial vehicle and therefore, given more information, the algorithm proposed by Cacace et al. can select which aerial vehicle needs to take off. In [59], the authors also propose a scheme that seeks to establish an effective cooperation between a human and a team of robots during navigation.
The element of heterogeneity in a robot team is even evident in the earlier works of MRS. The framework ALLIANCE, proposed by Parker [30], supports and exploits the inherent differences in a team of diverse robot platforms. This is achieved by tailoring the motivational behaviour model and behaviour sets according to the capabilities of the robots. Similarly, Gerkey and Matarić [60] propose a market-based planner system named MURDOCH for task allocation in a robotic team. In MURDOCH task messages are published over a network with a subject relating to the capabilities required from a robot to perform that task, leaving only those capable robots to subscribe to these messages. In the framework ASyMTRe [61], Tang and Parker propose an algorithm that decomposes a general task into sub-tasks according to pre-defined schema which reflect the different capabilities of the robots. These schema are then connected to assign the subtasks to the team members. Furthermore, Jeon et al. [26] propose a scheme with leader/follower roles for robots which are meant to survey an area and act in the event that an intruder is detected. These roles are defined according to the ability of the robot. A mission is decomposed into tasks, which are then assigned to the robots whose capabilities make them the most adequate to perform them. Similarly, in [58] and [62] we see search and rescue MRSs, where robots with different capabilities, such as to provide aerial views [58], or extinguish fires and transporting victims [62], are assigned to specific tasks. Additionally, the element of heterogeneity is strong in ground-to-aerial vehicle cooperation as seen in [63]-[65].

\section{F. Adversarial Environment}

In 1997 RoboCup was founded with the aim of promoting research in robotics and artificial intelligence. This competition has shifted some of the research attention onto MRS in adversarial environments, such as those found in soccer competitions or battlefields. In [66] Weigel et al. propose a novel approach that tracks the ball and the adversarial players, and at the same time it strategically coordinates the team. In the mission to win against the other team, a specific role requiring a number of skills, is assigned to each robot such that, each team member can then adopt adequate behaviour from a behaviour set. A similar approach is also adopted in [67], where Browning et al. propose a hierarchical architecture of Skills, Tactics and Plays to execute low-level actions, decide on the skills to use, and coordinate the activity among the team. Furthermore, this problem has also been solved by using reinforcement learning techniques, as has been done in [68] and [69]. Alternatively, MRS have also been involved in different adversarial environments, such as in battlefields. For instance, in [70] Zhang et al. use a genetic algorithm to enable robots to learn not to enter their adversaries' defence region, where they may be "killed". Similarly, this problem has also been studied and solved in [71] and [72]. Using a somewhat different approach, in [73] the authors look at the adversarial environment as being inherent to an auction system, where each member bids for a task to perform in a mission. 


\section{CONCLUSIONS}

In this paper, we have reviewed and classified prominent and recent works according to six main application domains in the field of MRS. From this survey of works it is possible to draw up a number of conclusions about the current challenges facing this field and its research direction. Primarily, it is clear that in most application domains, limited communication among the team members, is one of the major difficulties that needs to be solved in order to design an efficient and robust system. Another pressing issue, especially in multi-UAV applications, seems to be energy consumption and limited battery life. Researchers are proposing methods on how this constraint is incorporated in the solution of a particular task. Finally, the introduction of a human-in-the-loop is another promising research avenue to address a number of challenging real-life scenarios. However, this creates new challenges of its own, particularly due to the complex interaction that exists between a human being and a machine, which is naturally more pronounced in a humanto-many-machines applications.

\section{REFERENCES}

[1] A. Farinelli, L. Iocchi, D. Nardi, and A. Multirobot, "Multirobot Systems : A Classification Focused on Coordination," IEEE Transactions on Systems, Man, and Cybernetics Part B: Cybernetics, vol. 34, no. 5, pp. 2015-2028, 2004

[2] L. E. Parker, "Multiple Mobile Robot Systems," in Handbook of Robotics, 2008, ch. 40, pp. $921-941$.

[3] Y. Cai and S. X. Yang, "A Survey on Multi-robot Systems," in World Automation Congress 2012, 2012, pp. 1-6.

[4] Y. Zhi, N. Jouandeau, and A. A. Cherif, "A Survey and Analysis of Multi-Robot Coordination," International Journal of Advanced Robotic Systems, INTECH, vol. 10, no. 12, p. 399, 2013.

[5] Y. Cao, W. Yu, W. Ren, and G. Chen, "An overview of recent progress in the study of distributed multi-agent coordination," IEEE Transactions on Industrial Informatics, vol. 9, no. 1, pp. 427-438, 2013.

[6] B. P. Gerkey and M. J. Mataric, "A formal analysis and taxonomy of task allocation in multi-robot systems," International Journal of Robotics Research, vol. 23, no. 9, pp. 939-954, 2004.

[7] A. Khamis, A. Hussein, and A. Elmogy, Multi-robot Task Allocation: A Review of the State-of-the-Art. Springer, 2015.

[8] M. Bernardine Dias, R. Zlot, N. Kalra, and A. Stentz, "Market-based multirobot coordination: A survey and analysis," in Proceedings of the IEEE, vol. 94, no. 7, 2006, pp. 1257-1270.

[9] M. Brambilla, E. Ferrante, and M. Birattari, "Swarm robotics : A review from the swarm engineering perspective," in Swarm Intelligence, 2012, vol. 7, no. 1, pp. 1-41.

[10] M. Dorigo, D. Floreano, L. M. Gambardella, F. Mondada, S. Nolfi, T. Baaboura, M. Birattari, M. Bonani, M. Brambilla, A. Brutschy, D. Burnier, A. Campo, A. L. Christensen, A. Decugniere, G. Di Caro, F. Ducatelle, E. Ferrante, A. Forster, J. M. Gonzales, J. Guzzi, V. Longchamp, S. Magnenat, N. Mathews, M. Montes De Oca, R. O'Grady, C. Pinciroli, G. Pini, P. Retornaz, J. Roberts, V. Sperati, T. Stirling, A. Stranieri, T. Stutzle, V. Trianni, E. Tuci, A. E. Turgut, and F. Vaussard, "Swarmanoid: A novel concept for the study of heterogeneous robotic swarms," IEEE Robotics and Automation Magazine, vol. 20, no. 4, pp. 60-71, 2013.

[11] B. T. Champion and M. A. Joordens, "Underwater swarm robotics review," 10th System of Systems Engineering Conference, SoSE 2015, pp. 111-116, 2015.

[12] P. Agrawal, "A Review on Multi Robot Cooperation Using Bio Inspired Neural Networks," International Journal of Soft Computing, Mathematics and Control (IJSCMC), vol. 2, no. 4, pp. 15-24, 2013.

[13] M. A. Goodrich and A. C. Schultz, "Human-Robot Interaction: A Survey," Foundations and Trends in Human-Computer Interaction, vol. 1, no. 3, pp. 203-275, 2007.
[14] J. Y. C. Chen and M. J. Barnes, "Human Agent Teaming for Multirobot Control : A Review of Human Factors Issues," IEEE Transactions on Human-Machine Systems, vol. 44, no. 1, pp. 13-29, 2014.

[15] W. Guanghua, L. Deyi, G. Wenyan, and J. Peng, "Study on Formation Control of Multi-Robot Systems," in 3rd International Conference on Intelligent System Design and Engineering Applications, 2013, pp. $1335-1339$.

[16] Y. Mao, G. G. Yan, and Y. T. Tian, "A review of studies in flocking for multi-robot system," in International Conference on Computer, Mechatronics, Control and Electronic Engineering, CMCE 2010, vol. 4, 2010, pp. 28-31.

[17] D. Portugal and R. Rocha, "A Survey on Multi-robot Patrolling Algorithms," in Technological Innovation for Sustainability: Second IFIP WG 5.5/SOCOLNET Doctoral Conference on Computing, Electrical and Industrial Systems, DoCEIS 2011, L. M. Camarinha-Matos, Ed. Springer Berlin Heidelberg, 2011, pp. 139-146.

[18] Y. Liu and G. Nejat, "Robotic urban search and rescue: A survey from the control perspective," pp. 147-165, 2013.

[19] D. Cook, A. Vardy, and R. Lewis, "A survey of AUV and robot simulators for multi-vehicle operations," in IEEE/OES Autonomous Underwater Vehicles, AUV 2014, 2014, pp. 1 - 8.

[20] J.-H. Son and H.-S. Ahn, "Cooperative Reinforcement Learning: Brief Survey and Application to Bio-insect and Artificial Robot Interaction," in Science And Technology, 2008, pp. 71-76.

[21] A. Stancovici, M. V. Micea, and V. Cretu, "Cooperative Positioning System for Indoor Surveillance Applications," in International Conference on Indoor Positioning and Indoor Navigation, (IPIN), no. 1, 2016, pp. 1-7.

[22] R. Mendonça, M. M. Marques, F. Marques, A. Lourenço, E. Pinto, P. Santana, F. Coito, V. Lobo, and J. Barata, "A Cooperative MultiRobot Team for the Surveillance of Shipwreck Survivors at Sea," in MTS/IEEE Monterey OCEANS, 2016, pp. 2-7.

[23] N. Nigam, S. Bieniawski, I. Kroo, and J. Vian, "Control of multiple UAVs for persistent surveillance: Algorithm and flight test results," IEEE Transactions on Control Systems Technology, vol. 20, no. 5, pp. 1236-1251, 2012.

[24] E. Stump and N. Michael, "Multi-robot persistent surveillance planning as a vehicle routing problem," in IEEE International Conference on Automation Science and Engineering, 2011, pp. 569-575.

[25] A. Pustowka and E. F. Caicedo, "Market-based task allocation in a multi-robot surveillance system," in Brazilian Robotics Symposium and Latin American Robotics Symposium, SBR-LARS 2012, 2012, pp. 185-189.

[26] S. Jeon, M. Jang, S. Park, D. Lee, Y. J. Cho, and J. Kim, "Task allocation strategy of heterogeneous multi-robot for indoor surveillance," in 8th International Conference on Ubiquitous Robots and Ambient Intelligence, 2011, pp. 169-173.

[27] J. Gregory, J. Fink, E. Stump, J. Twigg, J. Rogers, N. Fung, and S. Young, "Application of Multi-Robot Systems to Disaster-Relief Scenarios with Limited Communication," Springer Tracts in Advanced Robotics, vol. 113, pp. 639-653, 2016.

[28] C. Archetti, D. Feillet, A. Hertz, and M. G. Speranza, "The capacitated team orienteering and profitable tour problems," Journal of the Operational Research Society, vol. 60, no. 6, pp. 831-842, 2009.

[29] M. A. Gutierrez, S. Nair, R. E. Banchs, L. F. D. H. Enriquez, A. I. Niculescu, and A. Vijayalingam, "Multi-robot collaborative platforms for humanitarian relief actions," in IEEE Region 10 Humanitarian Technology Conference, (R10-HTC), 2016.

[30] L. E. Parker, "ALLIANCE : An Architecture for Fault Tolerant Multirobot Cooperation," IEEE Transactions on Robotics and Automation, vol. 14 , no. 2, pp. 220-240, 1998.

[31] M. Schneider-Fontán and M. J. Matarić, "Territorial multi-robot task division," IEEE Transactions on Robotics and Automation, vol. 14, no. 5, pp. 815-822, 1998.

[32] A. Lein and R. T. Vaughan, "Adapting to non-uniform resource distributions in robotic swarm foraging through work-site relocation," IEEE/RSJ International Conference on Intelligent Robots and Systems, (IROS), pp. 601-606, 2009.

[33] M. S. Couceiro, R. P. Rocha, C. M. Figueiredo, J. M. A. Luz, and N. M. Fonseca Ferreira, "Multi-Robot Foraging based on Darwin's Survival of the Fittest," in IEEE/RSJ International Conference on Intelligent Robots and Systems, 2012, pp. 801-806.

[34] F. J. Mendiburu, M. R. A. Morais, and A. M. N. Lima, "Behavior co- 
ordination in multi-robot systems," in IEEE International Conference on Automatica (ICA-ACCA), 2016, pp. 1-7.

[35] D. Sakai, H. Fukushima, and F. Matsuno, "Flocking for Multirobots Without Distinguishing Robots and Obstacles," IEEE Transactions on Control Systems Technology, pp. 1-9, 2016.

[36] D. Gu and Z. Wang, "Leader-follower flocking: Algorithms and experiments," IEEE Transactions on Control Systems Technology, vol. 17, no. 5, pp. 1211-1219, 2009.

[37] H. M. La, R. Lim, and W. Sheng, "Multirobot cooperative learning for predator avoidance," IEEE Transactions on Control Systems Technology, vol. 23, no. 1, pp. 52-63, 2015.

[38] S. K. Lee, D. Kim, D. S. Shin, T. Jang, and J. Mclurkin, "Distributed Deformable Configuration Control for Multi-Robot Systems," in IEEE/RSJ International Conference on Intelligent Robots and Systems (IROS), 2016, pp. 5347 - 5354.

[39] X. Wang, W. Ni, and X. Wang, "Leader-following formation of switching multirobot systems via internal model," IEEE Transactions on Systems, Man, and Cybernetics, Part B: Cybernetics, vol. 42, no. 3, pp. 817-826, 2012.

[40] Y. Wang, L. Cheng, Z.-g. Hou, and M. Tan, "Optimal Formation of Multi-Robot Systems Based on a Recurrent Neural Network," IEEE Transactions on Neural Networks and Learning Systems, vol. 27, no. 2, pp. 1-11, 2016.

[41] R. Phanichnitinon, T. Hemwarangkoon, J. Polvichai, T. Boonpromma, and K. Jarutekumporn, "Multi modular robots maneuver for geometry formation control," in IEEE 7th International Workshop on Computational Intelligence and Applications, (IWCIA) 2014 - Proceedings, 2014, pp. 195-200.

[42] S. M. Lee, H. Kim, H. Myung, and X. Yao, "Cooperative coevolutionary algorithm-based model predictive control guaranteeing stability of multirobot formation," IEEE Transactions on Control Systems Technology, vol. 23, no. 1, pp. 37-51, 2015.

[43] J. Banfi, A. Q. Li, N. Basilico, I. Rekleitis, and F. Amigoni, "Asynchronous multirobot exploration under recurrent connectivity constraints," IEEE International Conference on Robotics and Automation, pp. 5491-5498, 2016.

[44] K. Cesare, R. Skeele, S.-h. Yoo, Y. Zhang, and G. Hollinger, "MultiUAV Exploration with Limited Communication and Battery," IEEE International Conference on Robotics and Automation (ICRA), no. 1, pp. 2230-2235, 2015.

[45] J. D. Hoog, S. Cameron, and A. Visser, "Autonomous Multi-Robot Exploration in Communication-Limited Environments," in 11th Conference Towards Autonomous Robotic Systems, 2010.

[46] U. Jain, R. Tiwari, S. Majumdar, and S. Sharma, "Multi Robot Area Exploration Using Circle Partitioning Method," in International Symposium on Robotics and Intelligent Sensors, vol. 41, 2012, pp. 383-387.

[47] F. Cabrera-Mora and J. Xiao, "A flooding algorithm for multirobot exploration," IEEE Transactions on Systems, Man, and Cybernetics, Part B: Cybernetics, vol. 42, no. 3, pp. 850-863, 2012.

[48] P. Brass, A. Gasparri, F. Cabrera-mora, and J. Xiao, "Multi-robot Tree and Graph Exploration," in IEEE International Conference on Robotics and Automation, 2009, pp. 2332-2337.

[49] L. E. Parker, "Multi-Robot Team Design for Real-World Applications," in Distributed Autonomous Robotic Systems 2. Springer Japan, 1996, no. 1 , ch. 4, pp. 91-102.

[50] M. J. Matarić, M. Nilsson, and K. T. Simsarian, "Cooperative MultiRobot Box-Pushing," in IEEE/RSJ International Conference onIntelligent Robots and Systems 95. 'Human Robot Interaction and Cooperative Robots', no. Figure 1, 1995, pp. 556-561.

[51] A. Ghosh, A. Ghosh, A. Konar, S. Member, and R. Janarthanan, "Multi-Robot Cooperative Box-pushing Problem Using Multi-objective Particle Swarm Optimization Technique," in World Congress on Information and Communication Technologies, (WICT), 2012, pp. 272-277.

[52] R. Brown and J. Jennings, "A pusher/steerer model for strongly cooperative mobile robot manipulation," in IEEE/RSJ International Conference on Intelligent Robots and Systems. Human Robot Interaction and Cooperative Robots, 1995, pp. 562-568.

[53] D. Sieber, F. Deroo, and S. Hirche, "Formation-based approach for multi-robot cooperative manipulation based on optimal control design," in IEEE International Conference on Intelligent Robots and Systems, 2013, pp. 5227-5233.

[54] W. Wan, R. Fukui, M. Shimosaka, T. Sato, and Y. Kuniyoshi, "Cooperative manipulation with least number of robots via robust caging," in IEEE/ASME International Conference on Advanced Intelligent Mechatronics, AIM, 2012, pp. 896-903.

[55] A. Zambelli Bais, S. Erhart, L. Zaccarian, and S. Hirche, "Dynamic load distribution in cooperative manipulation tasks," in IEEE/RSJ International Conference on Intelligent Robots and Systems (IROS), 2015, pp. 2380-2385.

[56] A. Amanatiadis, C. Henschel, B. Birkicht, B. Andel, K. Charalampous, I. Kostavelis, R. May, and A. Gasteratos, "AVERT : An Autonomous Multi-Robot System for Vehicle Extraction and Transportation," in IEEE International Conference on Robotics and Automation (ICRA), 2015, pp. 1662-1669.

[57] A. Rossi, M. Staffa, and S. Rossi, "Supervisory Control of Multiple Robots through Group Communication," IEEE Transactions on Cognitive and Developmental Systems, vol. 8920, no. c, pp. 1-12, 2016.

[58] J. Cacace, A. Finzi, and V. Lippiello, "Implicit Robot Selection for Human Multi-Robot Interaction in Search and Rescue Missions," in 25th IEEE International Symposium on Robot and Human Interactive Communication (RO-MAN), 2016, pp. 803 - 808.

[59] J. Saez-pons, L. Alboul, and J. Penders, "Experiments in Cooperative Human Multi-Robot Navigation," in IEEE International Conference on Robotics and Automation (ICRA), 2011, pp. 1 - 4 .

[60] B. P. Gerkey and M. J. Mataric, "Sold !: Auction Methods for Multirobot Coordination," IEEE Transactions on Robotics and Automation, vol. 18, no. 5, pp. 758-768, 2002.

[61] F. Tang and L. E. Parker, "ASyMTRe : Automated Synthesis of Multi-Robot Task Solutions through Software Reconfiguration," in IEEE International Conference on Robotics and Automation, no. April, Barcelona, Spain, 2005, pp. 1501 - 1508.

[62] I. Budinská and Š. Havlik, "Task allocation within a heterogeneous multi-robot system," 28th International Conference Cybernetics and Informatics, 2016.

[63] A. Dewan, A. Mahendran, N. Soni, and K. M. Krishna, "Heterogeneous UGV-MAV exploration using integer programming," 2013 IEEE/RSJ International Conference on Intelligent Robots and Systems, pp. 5742-5749, 2013.

[64] P. Stegagno, M. Cognetti, L. Rosa, P. Peliti, and G. Oriolo, "Relative localization and identification in a heterogeneous multi-robot system," IEEE International Conference on Robotics and Automation, pp. 1857-1864, 2013

[65] E. H. C. Harik, F. Guinand, H. Pelvillain, F. Guérin, and J. F. Brethé, "A decentralized interactive architecture for aerial and ground mobile robots cooperation," in International Conference on Control, Automation and Robotics, ICCAR 2015, 2015, pp. 37-43.

[66] T. Weigel, J. S. Gutmann, M. Dietl, A. Kleiner, and B. Nebel, "CS Freiburg: Coordinating robots for successful soccer playing," IEEE Transactions on Robotics and Automation, vol. 18, no. 5, pp. 685699, 2002.

[67] B. Browning, J. Bruce, M. Bowling, and M. Veloso, "STP: skills, tactics, and plays for multi-robot control in adversarial environments," IEEE Journal of Control and Systems Engineering, vol. 219, no. 1, pp. 33-52, 2004

[68] P. Stone, R. S. Sutton, and G. Kuhlmann, "Reinforcement Learning for RoboCup-Soccer Keepaway," Adaptive Behavior, vol. 13, no. 3, pp. 165-188, 2005.

[69] S. Kalyanakrishnan, Y. Liu, and P. Stone, "Half Field Offense in RoboCup Soccer: A Multiagent Reinforcement Learning Case Study,' RoboCup 2006: Robot Soccer World Cup X, pp. 72-85, 2007.

[70] D. Zhang and L. Wang, "Target Topology Based Task Assignment for Multiple Mobile Robots in Adversarial Environments," pp. 5323-5328, 2007.

[71] N. Agmon, S. Kraus, and G. A. Kaminka, "Multi-robot perimeter patrol in adversarial settings," in Proceedings - IEEE International Conference on Robotics and Automation, 2008, pp. 2339-2345.

[72] Y. Shapira and N. Agmon, "Path planning for optimizing survivability of multi-robot formation in adversarial environments," IEEE International Conference on Intelligent Robots and Systems, vol. 2015Decem, pp. 4544-4549, 2015.

[73] K.-S. Hwang, J.-L. Lin, and H.-L. Huang, "Cooperative patrol planning of multi-robot systems by a competitive auction system," ICROSSICE International Koint Conference 2009, pp. 4359-4363, 2009. 